\title{
Light at Night Exposure Effects on Differentiation and Cell Cycle in the Rat Liver With Autonomic Nervous System Denervation
}

\author{
Mohammad Hossein Heidari ${ }^{1}$, Mona Zamanian Azodi ${ }^{*}$, Mohammad Reza Zali ${ }^{3}$, Zahra Akbari ${ }^{4}$ \\ ${ }^{1}$ Proteomics Research Center, Faculty of Paramedical Sciences, Shahid Beheshti University of Medical Sciences, Tehran, \\ Iran \\ ${ }^{2}$ Proteomics Research Center, Shahid Beheshti University of Medical Sciences, Tehran, Iran \\ ${ }^{3}$ Gastroenterology and Liver Diseases Research Center, Research Institute for Gastroenterology and Liver Diseases, Shahid \\ Beheshti University of Medical Sciences, Tehran, Iran \\ ${ }^{4}$ Laser Application in Medical Sciences Research Center, Shahid Beheshti University of Medical Sciences, Tehran, Iran
}

\section{*Correspondence to \\ Mona Zamanian Azodi, \\ Proteomics Research Center (PRC), Darband St., Qods Sq., Tehran, Iran. \\ Tel: +982122714248; \\ Email:mona.azodi@gmail.com}

Published online December 1, 2019

\begin{abstract}
Introduction: $\mathrm{N}$ Exposure to artificial light at night (LAN) affects human health and causes several functional modifications in the body. Obesity, diabetes, and hormonal changes are reported after exposure to LAN in humans. This study aims to highlight the critical features of biological terms that are affected in the liver of rats which received autonomic nervous system denervation.

Methods: The liver gene expression profiles of 8 male Wistar rats that received sympathetic plus parasympathetic hepatic denervation and were exposed to LAN from Gene Expression Omnibus (GEO) for 1 hour were compared with 5 controls. The significant differentially-expressed genes (DEGs) were screened by the protein-protein interaction (PPI) network analysis STRING database (an application of Cytoscape software). Also, CuleGO and CleuDedia, the 2 applications of Cytoscape software, were used for more analysis.

Results: Among 250 DEGs, 173 characterized genes with fold change more than 2 plus 100 added relevant genes were included in the PPI network. The analysis of the main connected component (MCC) led to introducing 15 hubs and 15 bottlenecks. CCT2, COPS7A, KAT2A, and ERCC1 were determined as hub-bottlenecks. Among hubs and bottlenecks, DHX15, KAT2A, CCT2, HSP90AB1, CCNE1, DHX16, LSM2, WEE1, CWC27, BAZ1B, RAB22A, DNM2, and DHX30 were linked to each other by various kinds of actions. СCT2 and KAT2A, the 2 hub-bottlenecks, were included in the interacted genes in the action map. Four classes of biological terms including negative regulation of non-motile cilium assembly, negative regulation of transforming growth factor beta activation, alpha-tubulin acetylation, and histamine-induced gastric acid secretion were identified as the critical biochemical pathways and biological processes.

Conclusion: Several essential functions such as differentiation, cell cycle, ribosome assembly, and splicing are affected by LAN in rat livers with autonomic nervous system denervation. Keywords: Light at night; Rat; Gene; Denervation; Network.
\end{abstract}

\section{Introduction}

Investigations indicate that exposure to artificial light at night (LAN) even during sleeping affects the body functions. Obesity after LAN is one of the well-known effects that is reported for humans. ${ }^{1}$ The incident of diabetes mellitus is another disorder that is discussed by several researchers. ${ }^{2}$ The effect of LAN on the circadian system and melatonin production in humans has been investigated and confirmed. ${ }^{3}$ In many cases, it is highlighted that LAN is associated with obesity, diabetes, and even the onset and promotion of cancer. Based on researches, autonomic nervous systems (ANS) including sympathetic and parasympathetic nervous systems are involved in LAN exposure. ${ }^{4}$

Understanding disease molecular mechanism is an attractive subject for biomarker and drug target discovery. There are several attempts to introduce a molecular perspective of LAN effects..$^{5-7}$ Recently, high numbers of methods such as proteomics and genomics have been used vastly in the biomedical sciences to monitor gene expression change in the case of diseases. These methods can provide large numbers of valuable data that are

Please cite this article as follows: Heidari MH, Zamanian Azodi M, Zali MR, Akbari Z. Light at night exposure effects on differentiation and cell cycle in the rat liver with autonomic nervous system denervation. J Lasers Med Sci. 2019;10(suppl 1):S43-S48. doi:10.15171/ jlms.2019.S8. 
associated with disorders. ${ }^{89}$ Analyzing a large amount of data is a difficult task without using bioinformatics and system biology sciences. ${ }^{10-12}$ Protein-protein interaction (PPI) network analysis is a useful method that is used to analyze such complex data for various types of diseases. In this approach, the genes or proteins interact in the interactome and based on the topological properties of the constructed network, the central genes will be determined. ${ }^{13,14}$ Several centrality parameters are available during network analysis and degree and betweenness are the 2 important ones. Degree refers to the number of connections that a node in the network makes with the other members of the network and betweenness is a function of the shortest paths which the node is related to them. ${ }^{15,16}$ The nodes with a high degree value and betweenness centrality are known as hubs and bottlenecks respectively. The common hubs and bottlenecks are called hub-bottlenecks that are known as crucial elements in the network. ${ }^{17}$

In the present study, gene expression change in male Wistar rats, investigated by Opperhuizen et $\mathrm{al},{ }^{4}$ was evaluated via PPI network analysis. The animals received liver sympathetic and parasympathetic denervation and were irradiated with LAN from 21:00 to 22:00 for a night. The critically affected genes were introduced and enriched via gene ontology.

\section{Methods}

The liver gene expression profiles of 5 male Wistar rats that received sympathetic plus parasympathetic hepatic denervation (Tx group) as control samples were obtained from Gene Expression Omnibus (GEO) under the title of GSE120980. Each series record in GEO was given a single and stable GEO accession number as GSExxx, while each sample record was allotted a GSMxxx accession number (https://www.ncbi.nlm.nih.gov/geo/info/overview.html). The rats were housed in the controlled 12:12/light:dark cycle (lights on at 7:00, lights off at 19:00). The similar profiles of 8 rats which received sympathetic plus parasympathetic hepatic denervation and were exposed to light at night from 21:00 to 22:00 and were sacrificed at 22:00 (TxLAN group) on the experimental day were selected from GSE120980. ${ }^{4}$ The profiles were statistically matched and among the top 250 significant DEGs, 173 individuals that were characterized and had fold change more than 2 were selected to be included in the PPI network construction. The selected DEGs and 100 relevant neighbors interacted via the STRING database by Cytoscape software v6.3.1. ${ }^{17}$ The network was analyzed by the Network analyzer application of Cytoscape software to identify the central nodes. After ignoring the added genes, $10 \%$ of top nodes based on the degree value and $\mathrm{BC}$ were introduced as hubs and bottlenecks. The common hubs and bottlenecks were determined as hubbottlenecks. Hubs and bottlenecks interacted in an action map by the CluPedia ${ }^{14}$ application of Cytoscape and the non-isolated ones were enriched by $\mathrm{CluGO}^{18}$ to find the related biochemical pathways and biological processes from KEGG and GO biological processes respectively. The description of the non-isolated hubs and bottlenecks in the action map was extracted from STRING ${ }^{19}$ and summarized.

\section{Results}

The gene expression profiles of 8 TxLAN samples and 5 Tx individuals are shown in Figure 1. Obviously, TxLAN profiles were affected by LAN and are characterized by higher level of gene expression relative to the $\mathrm{Tx}$ samples. The top 250 significant DEGs were targeted to be analyzed. Among them, 173 genes were recognized and they showed at least 2 fold expression change. The 173 selected DEGs plus 100 added relevant genes were included in the network. The network contained 25 isolated DEGs and a main connected component (MCC) which included 248 individuals (148 DEGs and 100 added genes). As it is shown in Figure 2, the nodes of the MCC are connected by 3149 undirected edges. The MCC was analyzed to determine the central nodes. Ignoring the added genes, the top $10 \%$ of the DEGs based on the degree value and betweenness centrality were identified as hubs and bottlenecks respectively (see Table 1). The common hubs and bottlenecks including CCT2, COPS7A, KAT2A, and ERCC1 were introduced as hub-bottlenecks. Since the hubs and bottlenecks are the critical nodes, the action map including expression, binding, activation, inhibition, reaction, ptmod, and catalysis was provided via CluPedia for the hubs and bottlenecks. As it is presented in Figure 3, DHX15, KAT2A, CCT2, HSP90AB1, CCNE1, DHX16, LSM2, WEE1, CWC27, BAZ1B, RAB22A, DNM2, and

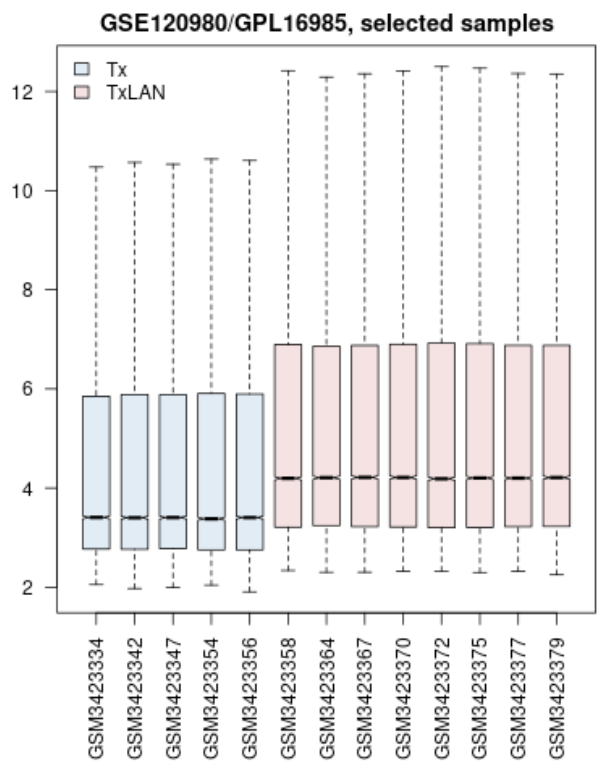

Figure 1. Box Plot Presentation of Gene Expression Profiles of the Samples Including 5 Controls (Tx) and 8 Treated (TxLAN). The distribution of the expressed genes in 4 quarters for each sample is illustrated. 


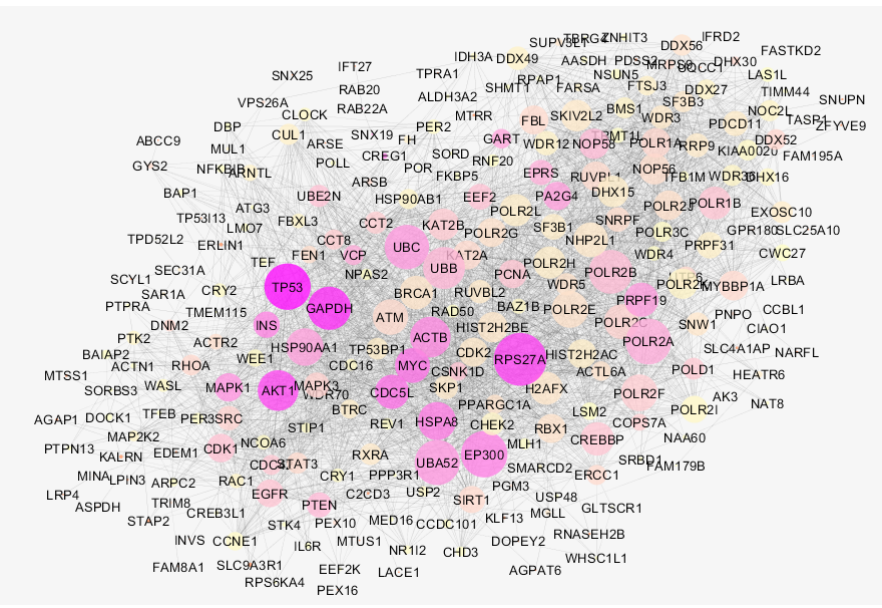

Figure 2. The Main Connected Component of the Network Including 248 Genes (148 DEGs and 100 Added Relevant Genes) and 3149 Edges. The nodes are shown based on degree values (bigger size refers to more degree values) and betweenness centrality (yellow to purple refers to the increment of $\mathrm{BC}$ ).

DHX30 are linked to the other nodes by various kinds of actions. CCT2 and KAT2A, the 2 hub-bottlenecks, are included in the interacted genes in the action map. The 13 interacted DEGs in the action map were selected for gene ontology analysis and the findings are shown in Figure 4.

Table 1. The Hubs and Bottlenecks of the Analyzed Network ${ }^{a}$

\begin{tabular}{|c|c|c|c|c|}
\hline Central Node & $\mathbf{R}$ & Display Name & Degree & BC \\
\hline \multirow{15}{*}{ Hub } & 1 & DHX15 & 54 & 0.125 \\
\hline & 2 & KAT2A & 50 & 0.281 \\
\hline & 3 & ССТ2 & 39 & 0.375 \\
\hline & 4 & $\mathrm{NOC} 2 \mathrm{~L}$ & 37 & 0.060 \\
\hline & 5 & ERCC1 & 35 & 0.281 \\
\hline & 6 & HSP90AB1 & 35 & 0.062 \\
\hline & 7 & POLR3C & 35 & 0.031 \\
\hline & 8 & CCNE1 & 32 & 0.062 \\
\hline & 9 & DHX16 & 30 & 0.062 \\
\hline & 10 & LSM2 & 30 & 0.000 \\
\hline & 11 & WEE1 & 30 & 0.062 \\
\hline & 12 & COPS7A & 29 & 0.375 \\
\hline & 13 & CWC27 & 29 & 0.000 \\
\hline & 14 & ARNTL & 28 & 0.030 \\
\hline & 15 & BAZ1B & 26 & 0.062 \\
\hline \multirow{15}{*}{ Bottleneck } & 1 & CREG1 & 8 & 1.000 \\
\hline & 2 & SNX19 & 2 & 0.750 \\
\hline & 3 & RAB22A & 2 & 0.500 \\
\hline & 4 & ССТ2 & 39 & 0.350 \\
\hline & 5 & COPS7A & 29 & 0.350 \\
\hline & 6 & DNM2 & 20 & 0.280 \\
\hline & 7 & DHX30 & 14 & 0.280 \\
\hline & 8 & KAT2A & 50 & 0.270 \\
\hline & 9 & ERCC1 & 35 & 0.270 \\
\hline & 10 & ARSB & 9 & 0.270 \\
\hline & 11 & MRPS9 & 16 & 0.270 \\
\hline & 12 & VPS26A & 4 & 0.260 \\
\hline & 13 & GYS2 & 9 & 0.260 \\
\hline & 14 & PNPO & 5 & 0.250 \\
\hline & 15 & PGM3 & 3 & 0.250 \\
\hline
\end{tabular}

a Hubs and bottlenecks are ranked based on degree and betweenness values respectively.
The negative regulation of non-motile cilium assembly, the negative regulation of transforming growth factor beta activation, alpha-tubulin acetylation, and histamineinduced gastric acid secretion were the 4 classes of biochemical pathways and biological processes that were affected by the non-isolated nodes in the action map. Descriptions for these 13 enriched DEGs were obtained from the STRING database (see Table 2).

\section{Discussion}

Since gene expression is a sensitive process for protecting and adapting the treated organism against condition changes, it can be expected that the gene expression profiles of the samples and the control rats will be different. However, the rate and kind of differences are important criteria. Results indicate that except ARNTL and NPAS2 which are down-regulated, the other DEGs are up-regulated. The gross expression changes in various genes refer to the effective interview by light at night on the treated rats, which is performed in the overexpression of huge numbers of genes. The clear difference in the gene expression pattern between the samples and controls is

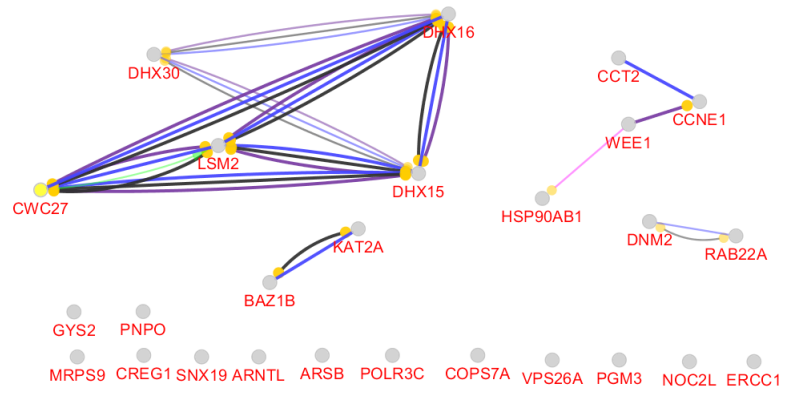

Figure 3. The Action Map of Hub and Bottleneck Nodes Including Binding (Blue), Activation (Green), Inhibition (Red), Catalysis (Purple), Reaction (Black), Expression (Yellow), And ptmod (Pink) Actions. The map is provided via the CluPedia application of Cytoscape software. 


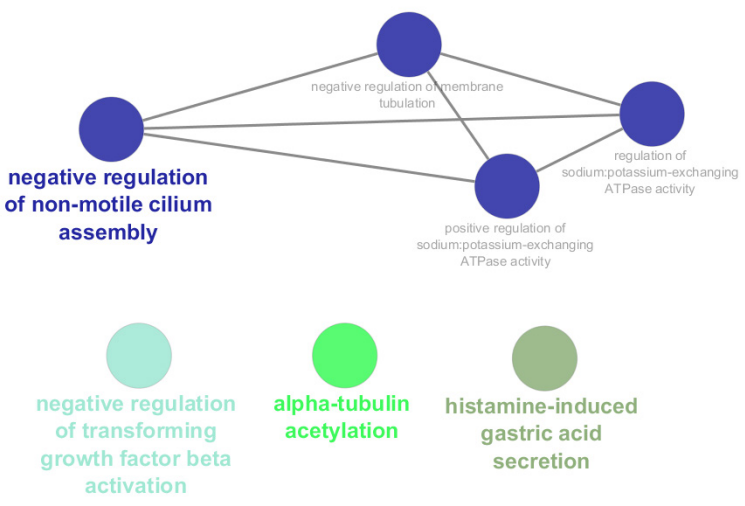

Figure 4. Biochemical Pathways and Biological Processes Related to the Non-isolated Hubs and Bottlenecks in the Action Map. The findings are obtained from KEGG and GO biological processes via the CluGO application of Cytoscape software.

shown in Figure 1. Differences are shown by 173 different DEGs that imply the use of a screening method for finding the more significant ones.

There are several pieces of evidence that network analysis is a useful method for evaluating large numbers of genes to identify the crucial ones. ${ }^{20}$ As it is depicted in Figure 2, the queried DEGs interact with each other to construct a scale-free network; however, the role of the added 100 significant genes in the promotion of interactions is prominent. Network analysis led to introducing 15 hubs and 15 bottlenecks among the 173 queried DEGs. Considering 4 hub-bottleneck genes, the numbers of favorable DEGs reduced to 26 ones. In the next step of screening, the action map showed that 13 DEGs among the 26 hubs and bottlenecks were involved in the regulating processes of each other. These central regulating DEGs can be considered as the critical genes which reveal the nature of changes after exposure to light at night in the treated rats. A complex including DHX15, DHS16, DHX30, CWC27, and LSM2 is formed in the action map. There are several kinds of connections between the members of this subnetwork. DHX is a member of this subnetwork; there is evidence that the members of the DHX family play roles in differentiation and carcinogenesis. ${ }^{21}$ The other element of this complex is CWC27 and retinal degeneration is attributed to the

Table 2. Descriptions of the Non-isolated Nodes in the Action Map Deriving From the STRING Database

\begin{tabular}{lll}
\hline $\mathbf{R}$ & Display Name & Description \\
\hline 1 & DHX15 & $\begin{array}{l}\text { Pre-mRNA-splicing factor ATP-dependent RNA helicase DHX15; It is involved in disassembly of spliceosomes after the release of } \\
\text { mature mRNA. }\end{array}$ \\
& $\begin{array}{l}\text { General control of amino acid synthesis protein 5-like 2; Functions as a histone acetyltransferase (HAT) to promote transcriptional } \\
\text { activation. In case of HIV-1 infection, it is recruited by the viral protein Tat. Regulates Tat's transactivating activity and may help inducing }\end{array}$
\end{tabular}
chromatin remodeling of proviral genes.

3 СCT2 the BBS/CCT complex, it may play a role in the assembly of BBSome, a complex involved in ciliogenesis regulating transports vesicles to the cilia. It is Known to play a role, in vitro, in the folding of actin and tubulin; Chaperonins maintenance and proper regulation of specific target proteins involved for instance in cell cycle control and signal transduction. It undergoes a functional cycle that is linked to its ATPase activity.

5 CCNE1 G1/S-specific cyclin-E1; Essential for the control of the cell cycle at the G1/S (start) transition; it belongs to the cyclin family. Cyclin E subfamily.

6 DHX16

Putative pre-mRNA-splicing factor ATP-dependent RNA helicase DHX16; Probable ATP-binding RNA helicase involved in pre-mRNA splicing; it belongs to the DEAD box helicase family.

LSM2 homolog, U6 small nuclear RNA associated (S. cerevisiae); it binds specifically to the 3'-terminal U-tract of U6 snRNA. It may be involved in pre-mRNA splicing; it belongs to the snRNP Sm proteins family.

WEE1 G2 checkpoint kinase; it acts as a negative regulator of entry into mitosis (G2 to $M$ transition) by protecting the nucleus from cytoplasmically activated cyclin B1-complexed CDK1 before the onset of mitosis by mediating phosphorylation of CDK1 on 'Tyr-15'.

CWC27 spliceosome-associated protein homolog (S. cerevisiae); PPlases accelerate the folding of proteins; it belongs to the cyclophilintype PPlase family.

Williams-Beuren syndrome chromosomal region 10 protein; Atypical tyrosine-protein kinase that plays a central role in chromatin remodeling and acts as a transcription regulator. It is involved in DNA damage response by phosphorylating 'Tyr-142' of histone $\mathrm{H} 2 \mathrm{AX}$ (H2AXY142ph). H2AXY142ph plays a central role in DNA repair and acts as a mark that distinguishes between apoptotic and repair responses to genotoxic stress. TF from early endosomes to recycling endosomes. It is required for NGF-mediated endocytosis of NTRK1, and subsequent neurite outgrowth. It binds GTP and GDP and has low GTPase activity.

Dynamin 2; Microtubule-associated force-producing protein involved in producing microtubule bundles and able to bind and hydrolyze GTP. It plays a role in the regulation of neuron morphology, axon growth and formation of neuronal growth cones (by similarity). It plays an important role in vesicular trafficking processes, in particular, endocytosis. It is involved in cytokinesis. It regulates the maturation of apoptotic cell corpse-containing phagosomes by recruiting PIK3C3 to the phagosome membrane (by similarity).

Putative ATP-dependent RNA helicase DHX30; it plays an important role in the assembly of the mitochondrial large ribosomal subunit. It is required for the optimal function of the zinc-finger antiviral protein ZC3HAV1 (by similarity). Associates with mitochondrial DNA DEAH-box helicases 
dysregulation of CWC27.22

Gene ontology revealed that the negative regulation of non-motile cilium assembly, the negative regulation of transforming growth factor beta activation, alphatubulin acetylation, and histamine-induced gastric acid secretion are the 4 major terms which are related to the 13 critical DEGs. It is reported that exposure to LAN mostly affects the body's metabolism. Obesity, diabetes and other aspects of liver function are the affected processes after LAN exposure. ${ }^{23,24}$ In this study, these features of function alterations are not highlighted. It seems that hepatic sympathetic and parasympathetic denervation is responsible for these events. Here the different kinds of alterations which are not related to the autonomic neuro system are presented. As it is tabulated in Table 2, the essential cell functions such as protein folding, cell cycle, splicing, apoptosis, neuron growth, protein transport, ribosome assembly, DNA repair, endocytosis, and antiviral activity are identified as dysregulated processes after LAN exposure.

In the previous study, alteration in the levels of such hormones as melatonin, cortisol, and corticosterone in the body after LAN exposure was reported. It is clear that the hormonal changes are associated with gross function alteration in organisms. Studies indicate that sleep, metabolism, locomotor activity, body temperature, and the circadian system can be affected by LAN exposure. It seems that these effects of light at night irradiation are directly related to the ANS. ${ }^{4,25,26}$ On the other hand, in the case of sympathetic and parasympathetic denervation, alterations in the ANS-related criteria have attracted the attention of scientists and the non-related ones have not been considered. Although other functions such as certain glands or tissues were studied after LAN exposure, the investigated criteria were indirectly under the effect of ANS.

In this study, a new perspective of LAN effects on organisms is presented. The essential functions are vital for the growth and survival of organisms. These findings not only reflect the effect of LAN but present the critical features of processes among the large numbers of biochemical pathways and biological processes. However, based on the system biology concept, the complete separation of alteration in the 2 distinct groups (ANS dependent and non-dependent) is not possible.

\section{Conclusion}

Rat livers with ANS denervation are sensitive to LAN exposure. Some vital functions like differentiation, cell cycle, ribosome assembly, and splicing are affected by LAN irradiation, indicating the importance of time and pattern of artificial light exposure in the human lifestyle.

\section{Ethical Considerations}

Not applicable.

\section{Conflict of Interests}

The authors declare no conflict of interest.

\section{Acknowledgment}

Shahid Beheshti University of Medical Sciences supports this research.

\section{References}

1. Park YM, White AJ, Jackson CL, Weinberg CR, Sandler DP. Association of Exposure to Artificial Light at Night While Sleeping With Risk of Obesity in Women. JAMA Intern Med. 2019;179(8):1061-71. doi: 10.1001/ jamainternmed.2019.0571.

2. Obayashi K, Yamagami Y, Kurumatani N, Saeki K. Bedroom lighting environment and incident diabetes mellitus: a longitudinal study of the HEIJO-KYO cohort. Sleep Med. 2019. doi:10.1016/j.sleep.2019.07.006.

3. Xiang S, Dauchy RT, Hoffman AE, Pointer D, Frasch T, Blask DE, et al. Epigenetic inhibition of the tumor suppressor ARHI by light at night-induced circadian melatonin disruption mediates STAT 3-driven paclitaxel resistance in breast cancer. J Pineal Res. 2019;67(2):e12586. doi: 10.1111/jpi.12586.

4. Opperhuizen A-L, Foppen E, Jonker M, Wackers P, Van Faassen M, van Weeghel M, et al. Effects of light-at-night on the rat liver-a role for the autonomic nervous system. Front Neurosci. 2019;13:647. doi: 10.3389/fnins.2019.00647.

5. Desouhant E, Gomes E, Mondy N, Amat I. Mechanistic, ecological, and evolutionary consequences of artificial light at night for insects: review and prospective. Entomol Exp Appl. 2019;167(1):37-58. doi: 10.1111/eea.12754.

6. Durrant J, Green MP, Jones TM. Dim artificial light at night reduces the cellular immune response of the black field cricket, Teleogryllus commodus. Insect Sci. 2019. doi: 10.1111/1744-7917.12665.

7. Nagai N, Ayaki M, Yanagawa T, Hattori A, Negishi K, Mori $\mathrm{T}$, et al. Suppression of Blue Light at Night Ameliorates Metabolic Abnormalities by Controlling Circadian Rhythms. Invest Ophthalmol Vis Sci. 2019;60(12):3786-93. doi: 10.1167/iovs.19-27195.

8. Pankow S, Martínez-Bartolomé S, Bamberger C, Yates JR. Understanding molecular mechanisms of disease through spatial proteomics. Curr Opin Chem Biol. 2019;48:19-25. doi: 10.1016/j.cbpa.2018.09.016.

9. Prohaska A, Racimo F, Schork AJ, Sikora M, Stern AJ, Ilardo $\mathrm{M}$, et al. Human disease variation in the light of population genomics. Cell. 2019;177(1):115-31. doi: 10.1016/j.cell.2019.01.052.

10. Miles S, Portela M, Cyrklaff M, Ancarola ME, Frischknecht F, Durán R, et al. Combining proteomics and bioinformatics to explore novel tegumental antigens as vaccine candidates against Echinococcus granulosus infection. J Cell Biochem. 2019;120(9):15320-36. doi: 10.1002/jcb.28799.

11. Asgari E, Mofrad MR. Leveraging Biomedical and Healthcare Data. Deep Genomics and Proteomics: Language Model-Based Embedding of Biological Sequences and Their Applications in Bioinformatics. Elsevier; 2019. p. 167-81. doi:10.1016/B978-0-12-809556-0.00012-5.

12. Gomez-Varela D, Barry AM, Schmidt M. Proteome-based systems biology in chronic pain. J Proteomics. 2019;190:1- 
11. doi: 10.1016/j.jprot.2018.04.004.

13. Rezaei Tavirani M, Rezaei Tavirani M, Vafaee R. Interaction network prediction and analysis of Anorexia Nervosa. Iran J Child Neurol. 2019;13(3):45-54. doi: 10.22037/ijcn. v13i3.18999.

14. Zamanian-Azodi M, Rezaei-Tavirani M. Investigation of health benefits of cocoa in human colorectal cancer cell line, HT-29 through interactome analysis. Gastroenterol Hepatol Bed Bench. 2019;12(1):67-73. doi: 10.22037/ghfbb. v0i0.1557.

15. Zadeh-Esmaeel MM, Rezaei-Tavirani M, Ahmadi NA, Vafae R. Evaluation of gene expression change in eosinophilic gastroenteritis. Gastroenterol Hepatol Bed Bench. 2019;12(3):239-45. doi: 10.22037/ghfbb.v12i3.1635.

16. Zamanian Azodi M, Rezaei-Tavirani M, Rezaei-Tavirani M. Identification of the key genes of autism spectrum disorder through protein-protein interaction network. Galen Med J. 2019;8:1367. doi: 10.31661/gmj.v8i0.1367.

17. Rezaei-Tavirani M, Rezaei Tavirani M, Zamanian Azodi M, Akbari Z, Hajimehdipoor H. Prediction of coffee effects in rats with healthy and NAFLD conditions based on proteinprotein interaction network analysis. Res J Pharmacogn. 2019;6(4):7-15. doi: 10.22127/rjp.2019.93500.

18. Karbalaei R, Allahyari M, Rezaei-Tavirani M, AsadzadehAghdaei H, Zali MR. Protein-protein interaction analysis of Alzheimers disease and NAFLD based on systems biology methods unhide common ancestor pathways. Gastroenterol Hepatol Bed Bench. 2018;11(1):27-33.

19. KhalKhal E, Rezaei-Tavirani M, Razzaghi MR, RezaeiTavirani S, Zali H, Rostami-Nejad M. The critical role of dysregulation of antioxidant activity and carbohydrate metabolism in celiac disease. Gastroenterol Hepatol Bed
Bench. 2019;12(4):340-7. doi: 10.22037/ghfbb.v12i4.1745.

20. Rezaei-Tavirani M, Rezaei-Tavirani M, Zamanian Azodi M, Razzaghi MR, Farshi HM. Evaluation of skin response after erbium: yttrium-aluminum-garnet laser irradiation: a network analysis approach. J Lasers Med Sci. 2019;10(3):194-99. doi: 10.22037/jlms.v10i3.24698.

21. Abdelhaleem M, Maltais L, Wain H. The human DDX and DHX gene families of putative RNA helicases. Genomics. 2003;81(6):618-22. doi: 10.1016/s0888-7543(03)00049-1.

22. Xu M, Xie YA, Abouzeid H, Gordon CT, Fiorentino A, Sun Z, et al. Mutations in the spliceosome component CWC27 cause retinal degeneration with or without additional developmental anomalies. Am J Hum Genet. 2017;100(4):592-604. doi: 10.1016/j.ajhg.2017.02.008.

23. Opperhuizen AL, Stenvers DJ, Jansen RD, Foppen E, Fliers E, Kalsbeek A. Light at night acutely impairs glucose tolerance in a time-, intensity-and wavelength-dependent manner in rats. Diabetologia. 2017;60(7):1333-43. doi: doi: 10.1007/s00125-017-4262-y.

24. Cailotto C, Lei J, van der Vliet J, van Heijningen C, van Eden CG, Kalsbeek A, et al. Effects of nocturnal light on (clock) gene expression in peripheral organs: a role for the autonomic innervation of the liver. PLoS One. 2009;4(5):e5650. doi: 10.1371/journal.pone.0005650.

25. Zhang L, Chen F, Cao J, Dong Y, Wang Z, Hu M, et al. Green light inhibits GnRH-I expression by stimulating the melatonin-G $\mathrm{n}$ IH pathway in the chick brain. $J$ Neuroendocrinol. 2017;29(5). doi: 10.1111/jne.12468.

26. Yang Y, Yu Y, Yang B, Zhou H, Pan J. Physiological responses to daily light exposure. Sci Rep. 2016;6:24808. doi: 10.1038/ srep24808. 